\title{
El rivaroxabán fue más efectivo y menos seguro que la enoxaparina para la prevención de trombosis venosa profunda
}

\section{Rivaroxaban was more effective and less safe than enoxaparin for deep vein trombosis prevention}

\section{Objetivo}

Comparar la eficacia y la seguridad de rivaroxaban por vía oral (VO) con la de enoxaparina subcutánea.

\section{Diseño y lugar}

Ensayo clínico (multicéntrico e internacional) de no inferioridad, controlado, aleatorizado y doble ciego, liderado por el King's College Hospital, Londres.

\section{Pacientes e intervención}

Fueron elegibles 8101 mayores de 40 años que habían sido internados por una enfermedad aguda no quirúrgica. Fueron asignados a dos grupos: 1) enoxaparina subcutánea (40 $\mathrm{mg} /$ día) durante $10 \pm 4$ días más placebo VO durante $35 \pm 4$ días; 2) placebo por vía subcutánea durante $10 \pm 4$ días y rivaroxaban (10 mg/día VO) durante $35 \pm 4$ días.

\section{Medición de resultados principales}

La medida de efectividad primaria fue la incidencia de trombosis venosa asintomática proximal o sintomática hasta el día diez (prueba de no inferioridad*) y hasta el día 35 (de superioridad); y el principal resultado de seguridad, la incidencia* de sangrado mayor o clínicamente significativo. El análisis fue por intención de tratar.

\section{Resultados principales}

Se resumen en la tabla 1.
Cohen A y col. N Engl J Med. 2013;368(6):513-23. Tabla 1: comparación de la eficacia y la seguridad de rivaroxaban y enoxaparina en pacientes internados por enfermedad aguda no quirúrgica.

\begin{tabular}{|c|c|c|c|c|}
\hline \multirow[t]{2}{*}{ Resultado } & \multirow[t]{2}{*}{ Día } & \multicolumn{2}{|c|}{$\begin{array}{l}\text { Número de eventos/expuestos } \\
\text { (incidencia acumuladaa) }\end{array}$} & \multirow[t]{2}{*}{$\underset{\text { (IC95\%) }}{\mathbf{R R}}$} \\
\hline & & Rivaroxaban & Enoxaparina & \\
\hline \multirow{2}{*}{$\begin{array}{l}\text { Trombosis venosa } \\
\text { proximal o sintomática } \\
\text { (evento primario) }\end{array}$} & 10 & $\begin{array}{c}78 / 2938 \\
(2,7 \%)\end{array}$ & $\begin{array}{c}82 / 2993 \\
(2,7 \%)\end{array}$ & $\begin{array}{c}0.97 \\
(0,71 \mathrm{a} 1,31)\end{array}$ \\
\hline & 35 & $\begin{array}{c}131 / 2967 \\
(4,4 \%)\end{array}$ & $\begin{array}{c}175 / 3057 \\
(5,7 \%)\end{array}$ & $\begin{array}{c}0,77 \\
(0,62 \text { a } 0,96)\end{array}$ \\
\hline \multirow[t]{2}{*}{$\begin{array}{l}\text { Sangrado clínicamente } \\
\text { significativo }\end{array}$} & 10 & $\begin{array}{c}11 / 3997 \\
(2,8 \%)\end{array}$ & $\begin{array}{c}49 / 4001 \\
(1,2 \%)\end{array}$ & $\begin{array}{c}2,3 \\
(1,63 \mathrm{a} 3,17)\end{array}$ \\
\hline & 35 & $\begin{array}{c}164 / 3997 \\
(4,1 \%)\end{array}$ & $\begin{array}{c}67 / 4001 \\
(1,7 \%)\end{array}$ & $\begin{array}{c}2.5 \\
(1,85 \text { a } 3,25)\end{array}$ \\
\hline
\end{tabular}

a Para el cálculo de la incidencia de eventos primarios fue usado como denominador el número de personas que recibieron el tratamiento completo, y para el de la incidencia de efectos adversos, a quienes recibieron al menos una vez la droga en estudio.

\section{Conclusiones}

El rivaroxabán mostró ser más eficaz y menos seguro que la enoxaparina cuando se los comparó durante 35 días para la prevención de TVP en pacientes con enfermedades agudas no quirúrgicas.

Fuente de financiamiento: Bayer HealthCare Pharmaceuticals (fabricante del rivaroxaban) y Janssen Research and Development.

\section{Comentario}

El rivaroxaban es un inhibidor directo del factor $X$ que comenzó a ser investigado por Bayer en 2008. Las primeras aprobaciones en EE.UU. (2011) fueron para la prevención prolongada de la trombosis venosa profunda (TVP) posoperatoria en pacientes sometidos a artroplastia y otras cirugías ortopédicas $^{1}$, extendiéndose luego para la prevención del accidente cerebrovascular en pacientes con fibrilación auricular, y del embolismo pulmonar y la TVP recurrente luego del tratamiento inicial.

Tiene una vida media de cinco a nueve horas, una alta unión a proteínas y no es dializable, por lo que no posee un antídoto útil, lo que disminuye la probabilidad que se logre una rápida co-rrección ${ }^{2}$ en pacientes sangrantes que lo vienen recibiendo, ha-llazgos coincidentes con los reportados por Cohen y col. . $^{3,4}$.

Si bien la mayoría de los aspectos metodológicos de esta investigación han sido prolijos, queda dudas respecto del dis- cordante tiempo de duración de ambos tratamientos y su posible vinculación con el resultado primario evaluado (reducción del riesgo de un evento combinado de trombosis) ${ }^{5}$.

Dado que aún no está probada la utilidad del rivaroxabán para la prevención de TVP, y lo que es más importante, su perfil de seguridad en pacientes internados por patología no quirúrgica, todavía no es una droga incluida en las guías para prevención de TVP'

\section{Conclusiones del comentador}

El rivaroxaban sería una opción útil para la prevención de TVP en pacientes con enfermedades agudas no quirúrgicas, aunque su costo y riesgos asociados a su uso todavía no avalan su uso masivo.

Federico Carini [ Unidad de Terapia Intensiva Del Hospital Italiano de Buenos Aires.federico.carini@hiba.org.ar ]

Carini F. El rivaroxabán fue más efectivo y menos seguro que la enoxaparina para la prevención de de trombosis venosa profunda. Evid Act Pract Ambul Oct-Dic 2013; 16(4):129. Comentado de: Cohen A y col. Rivaroxaban for thromboprophylaxis in acutely ill medical patients. N Engl J Med. 2013;368(6):513-23.

\section{Referencias}

1. Eriksson B y col. Rivaroxaban versus enoxaparin for thromboprophylaxis after hip arthroplasty. N Engl J Med. 2008;358(26):2765-75

2. No authors listed. Bleeding with dabigatran, rivaroxaban, apixaban. No antidote, and little clinical experience. Prescrire Int. 2013;22(139):155-9.

3. Cohen A y col. Rivaroxaban for thromboprophylaxis in acutely ill medical patients. N Engl J Med. 2013;368(6):513-23.

4. Alameddine R y col. Rivaroxaban for thromboprophylaxis in acutely ill medical patients. N Engl J Med. 2013;368(20):1944-5.

5. Derksen R y col. Rivaroxaban for thromboprophylaxis in acutely ill medical patients. N Engl J Med. 2013;368(20):1945.

6. Guyatt GH, AkI EA, Crowther M, Gutterman DD, Schuünemann HJ. Executive summary: Antithrombotic Therapy and Prevention of Thrombosis, 9th ed: American College of Chest Physicians Evidence-Based Clinical Practice Guidelines. Chest. 2012;141(2 Suppl):7S-47S. 